\title{
ROCKING RESPONSE OF RECTANGULAR RIGID BLOCKS UNDER RANDOM NOISE BASE EXCITATIONS
}

\author{
R. N. IYengaR ${ }^{\dagger}$ and C. S. MANOHAR \\ Department of Civil Engineering, Indian Institute of Science, Bangalore 560012, India
}

\begin{abstract}
The response of a rigid rectangular block resting on a rigid foundation and acted upon simultaneously by a horizontal and a vertical random white-noise excitation is considered. In the equation of motion, the energy dissipation is modeled through a viscous damping term. Under the assumption that the body does not topple, the steady-state joint probability density function of the rotation and the rotational velocity is obtained using the Fokker-Planck equation approach. Closed form solution is obtained for a specific combination of system parameters. A more general but approximate solution to the joint probability density function based on the method of equivalent non-linearization is also presented. Further, the problem of overturning of the block is approached in the framework of the diffusion methods for first passage failure studies. The overturning of the block is deemed incipient when the response trajectories in the phase plane cross the separatrix of the conservative unforced system. Expressions for the moments of first passage time are obtained via a series solution to the governing generalized Pontriagin-Vitt equations. Numerical results illustrative of the theoretical solutions are presented and their validity is examined through limited amount of digital simulations.
\end{abstract}

\section{INTRODUCTION}

The behavior of block-like structures which rock while resting on a moving base has been a subject of considerable technical interest [1-3]. This problem is of relevance in the seismic safety analysis of nuclear containment structures, dams and heavy equipment. In engineering seismology this problem is of interest from the point of view of characterizing the ground motion level through the study of overturned objects. The simplest of the mathematical models that has received notable attention in the past has been the planar rocking of rigid rectangular blocks under harmonic base motions. In this problem, the non-linearity arises not only in the load deflection characteristic but also in the dissipation of energy due to impacts. Recent studies on the response of this system have revealed the presence of a rich variety of non-linear resonances and even the possibility of the response becoming chaotic [4-6]. While rocking response under deterministic excitation has received considerable attention, the corresponding studies on stochastic behavior are sparse. A significant work on stochastic analysis has been by Spanos and Koh [7]. They have employed the statistical linearization technique to study the stochastic rocking of a rigid rectangular block on Winkler's foundation due to non-stationary foundation shaking. Koh [8] has further studied this problem through a digital simulation technique and has estimated the probability of no toppling. In the present study, the stochastic response of a rigid rectangular block resting on rigid foundation and acted upon simultaneously by a horizontal and a vertical stationary white-noise excitation is considered. The sliding of the block is assumed to be not possible. The dissipation of energy due to impacts is assumed to be not possible. The dissipation of energy due to impacts is approximately modeled through an equivalent viscous damping term. For small noise levels, it is assumed that the body does not topple. For this case the stationary response probability density function (pdf) is obtained by solving the governing Fokker-Planck equation. An exact solution is found to be possible only for a specific combination of system parameters. A general, but approximate, solution is also obtained using the method of equivalent non-linearization. The problem of the overturning of the body is posed as a first passage problem. The system is considered to have failed or overturned when the response trajectory in the phase plane crosses the separatrix of the undamped unforced motion. Expressions for the moments of the first passage time are obtained by solving the governing generalized Pontriagin-Vitt (GPV) equations using a series solution technique.

\footnotetext{
To whom correspondence should be addressed.
} 

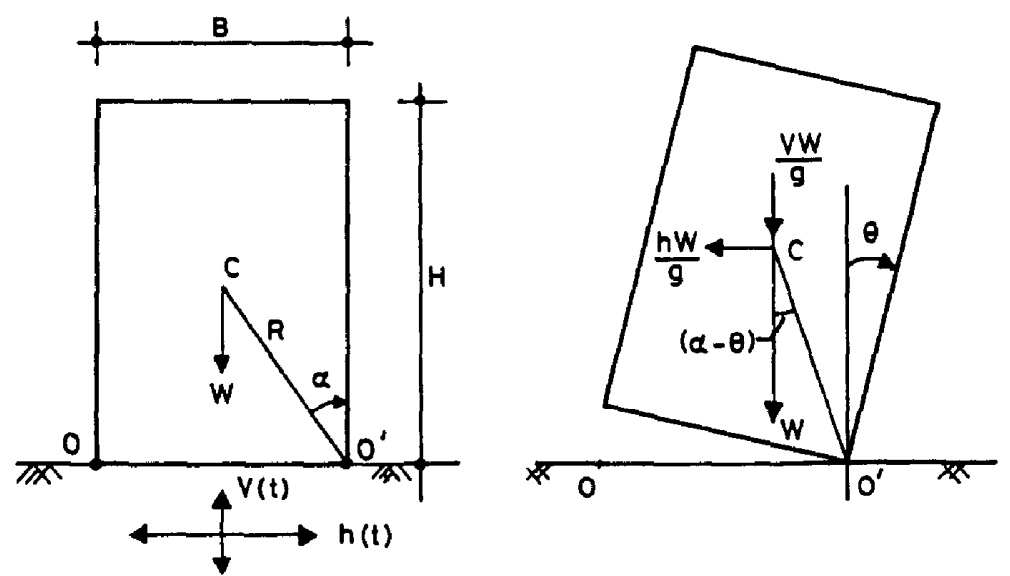

Fig. 1. Rocking rigid block.

\section{EQUATIONS OF MOTION}

The system under consideration is shown in Fig. 1. Under the action of the base accelerations $h(t)$ and $v(t)$ the block rocks about the corners $o$ and $o^{\prime}$. The equation of motion for the rotation $\Theta$ can be shown to be given by

$$
\begin{gathered}
I \ddot{\theta}+C \dot{\Theta}+W R \sin (\alpha \operatorname{sgn} \Theta-\Theta)[1+v(t) / g] \\
+W R \cos (\alpha \operatorname{sgn} \Theta-\Theta) h(t) / g=0 .
\end{gathered}
$$

Here dots denote the derivatives with respect to the time $t$, and $\operatorname{sgn}($.$) is the signum$ function. The angle $\Theta$ is taken to be positive when the block rocks about the corner $o$. Introducing the notations $\omega^{2}=(W R / I), 2 \eta \omega=(c / I), W_{1}(t)=V(t) / g$ and $W_{2}(t)=h(t) / g$ the above equation can be rewritten as

$$
\begin{aligned}
\ddot{\Theta} & +2 \eta \omega \dot{\Theta}+\omega^{2} \sin (\alpha \operatorname{sgn} \Theta-\Theta)\left[1+W_{1}(t)\right] \\
& +\omega^{2} \cos (\alpha \operatorname{sgn} \Theta-\Theta) W_{2}(t)=0
\end{aligned}
$$

In the ensuing analysis $W_{1}(t)$ and $W_{2}(t)$ are taken to be stationary Gaussian white-noise processes with the correlation functions given by $\left\langle W_{i}\left(t_{1}\right) W_{j}\left(t_{2}\right)\right\rangle=2 D_{i j} \delta\left(t_{2}-t_{1}\right), i, j=1$ and 2 . Here $\langle$. $\rangle$ denotes the expectation operator and $\delta($.$) is the Dirac's delta function. It$ may be noted that for the rectangular block one gets $\omega^{2}=0.75 \mathrm{~g} / R$. For the case in which no forces act on the system, the singular points of the governing equations of motion can easily be shown to be given by $(0,0)$ and $(0, \pm \alpha)$. Here the origin is found to be a stable singular point while the other two points are unstable. For the case of undamped free vibration the energy curves of the system are given by the equation

$$
h_{0}=0.5 \Theta^{2}+\omega^{2} \int_{0}^{\Theta} \sin (\alpha \operatorname{sgn} \xi-\xi) \mathrm{d} \xi .
$$

A phase portrait of the free vibration for the case of $\alpha=1.1, \omega=1$ and for different values of $h_{0}$ is shown in Fig. 2. It may be observed from this figure that the stable rocking motions about the origin are bounded by a separatrix. Thus it would be reasonable to take the region outside the separatrix to correspond to the unstable overturning of the block.

\section{STATIONARY STOCHASTIC RESPONSE}

For low levels of external noise, motions of the block initiated near the origin can be expected to result in rocking of the block without toppling. For this case one can further expect an existence of a stochastic steady state. In order to characterize such a response it may first be noted that the solution vector $[\Theta(t), \dot{\Theta}(t)]$ of equation (2) constitute a Markov 


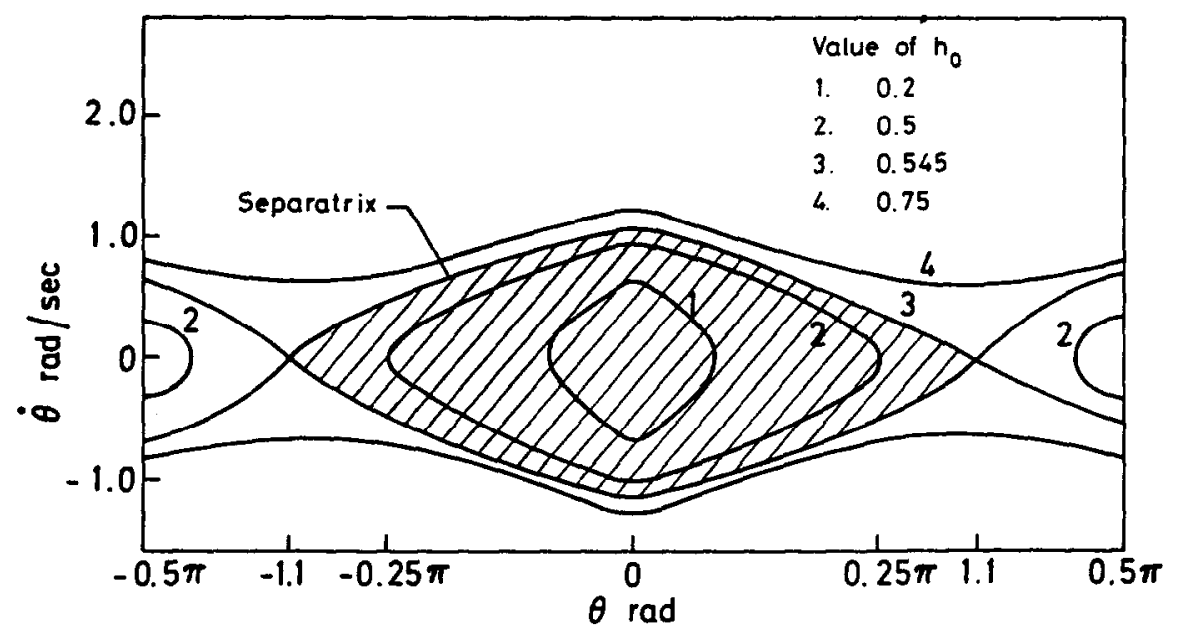

Fig. 2. Phase plane diagram for undamped free vibration $\omega=1, \alpha=1.1$.

vector process. Accordingly, the Fokker-Planck equation governing the transitional pdf, $p\left(\Theta, \dot{\Theta}, t \mid \Theta\left(t_{0}\right)=x, \dot{\Theta}\left(t_{0}\right)=y\right)$ can be derived as

$$
\begin{aligned}
\frac{\partial p}{\partial t}= & -\dot{\Theta} \frac{\partial p}{\partial \Theta}-\frac{\partial}{\partial \dot{\Theta}}\left\{\left[-2 \eta \omega \dot{\Theta}-\omega^{2} \sin (\alpha \operatorname{sgn} \Theta-\Theta)\right] p\right\} \\
& +f(\Theta) \frac{\partial^{2} p}{\partial \dot{\Theta}^{2}} \\
f(\Theta)= & \omega^{4}\left[D_{11} \sin ^{2}(\alpha \operatorname{sgn} \Theta-\Theta)+D_{22} \cos ^{2}(\alpha \operatorname{sgn} \Theta-\Theta)\right. \\
& \left.+D_{12} \sin ^{2}(\alpha \operatorname{sgn} \Theta-\Theta)\right] .
\end{aligned}
$$

For large times $\partial p / \partial t \rightarrow 0$, and the stationary solution can be obtained by solving the corresponding reduced Fokker-Planck equation. For this purpose, two cases can be considered separately.

Case (i). This is a special case in which $D_{11}=D_{22}$ and $D_{12}=0$. Here the diffusion coefficient $f(\Theta)$ reduces to a constant given by $f(\Theta)=\omega^{4} D_{11}$. In this case the stationary solution of equation (4) is obtained exactly as

$$
p_{s}(\Theta, \dot{\Theta})=\phi_{0} \exp \left[-\left(2 \eta / D \omega^{3}\right)\left\{0.5 \dot{\Theta}^{2}+\omega^{2} \int_{0}^{\theta_{1}} \sin (\alpha \operatorname{sgn} \xi-\xi) d \xi\right\}\right] .
$$

Here $\phi_{0}$ is the normalization constant which ensures that

$$
\int_{-0.5 \pi}^{0.5 \pi} \int_{-\infty}^{\infty} p_{s}(\Theta, \dot{\Theta}) \mathrm{d} \Theta \mathrm{d} \dot{\Theta}=1
$$

It follows from equation (6) that $\Theta$ and $\dot{\Theta}$ are independent in the steady state, and also that $\dot{\Theta}$ is Gaussian distributed, while the pdf of $\Theta$ is non-Gaussian.

Case (ii). This is a general case in which $D_{11} \neq D_{22}$ and $D_{12} \neq 0$. In this case the exact stationary solution of equation (4) is no longer obtainable. Thus, in the further analysis approximations are needed. In this study the method of equivalent non-linearization [9] is adopted for this purpose. Here the given equation

$$
-\dot{\Theta} \frac{\partial p}{\partial \Theta}-\frac{\partial}{\partial \Theta}\left[\left\{-2 \eta \omega \Theta-\omega^{2} \sin (\alpha \operatorname{sgn} \Theta-\Theta)\right\} p\right]+f(\Theta) \frac{\partial^{2} p}{\partial \dot{\Theta}^{2}}=0
$$

is replaced by an equivalent system

$$
-\dot{\Theta} \frac{\partial p}{\partial \Theta}-\frac{\partial}{\partial \dot{\Theta}}\left[\left\{-2 \eta \omega \Theta-\omega^{2} \sin (\alpha \operatorname{sgn} \Theta-\Theta)\right\} p\right]+\mu \frac{\partial^{2} p}{\partial \dot{\Theta}^{2}}=0
$$


Here $\mu$ is the equivalent parameter which in general can be a function of the energy of the system given by

$$
H_{0}=0.5 \dot{\Theta}^{2}+\omega^{2} \int_{0}^{\Theta} \sin (\alpha \operatorname{sgn} \xi-\xi) \mathrm{d} \xi .
$$

In the present analysis, however, for the sake of simplicity $\mu$ is taken to be a constant. Accordingly, the exact solution of equation (9) is now obtained as

$$
p_{s}(\Theta, \dot{\Theta})=\phi_{0} \exp \left[-(2 \eta \omega / \mu)\left\{0.5 \dot{\Theta}^{2}+\omega^{2} \int_{0}^{\Theta} \sin (\alpha \operatorname{sgn} \xi-\xi) \mathrm{d} \xi\right\}\right]
$$

where $\phi_{0}$ is the normalization constant satisfying equation (7). In order to determine the value of $\mu$ it may first be noted that the error of replacing equation (8) by equation (9) is given by the equation

$$
e=f(\Theta) \frac{\partial^{2} p}{\partial \dot{\Theta}^{2}}-\mu \frac{\partial^{2} p}{\partial \dot{\Theta}^{2}}
$$

The equivalent parameter $\mu$ is determined by minimizing the error

$$
E=\int_{-0.5 \pi}^{0.5 \pi} \int_{-\infty}^{\infty} e^{2}(\Theta, \dot{\Theta}) \mathrm{d} \Theta \mathrm{d} \dot{\Theta}
$$

with respect to $\mu$. Combining this criterion with equation (11) one obtains

$$
\mu=\frac{\int_{-0.5 \pi}^{0.5 \pi} f(\Theta) p_{s}^{2}(\Theta) \mathrm{d} \Theta}{\int_{-0.5 \pi}^{0.5 \pi} p_{s}^{2}(\Theta) \mathrm{d} \Theta} .
$$

Here $p_{s}(\Theta)$ is the marginal pdf of $\Theta$. It must be noted that $p_{s}(\Theta)$ itself is a function of $\mu$; this means that the above equation is a non-linear equation in $\mu$. From equation (11), it may be noted that $\Theta$ and $\dot{\Theta}$ are independent in this case also and again $\dot{\Theta}$ is normally distributed while $\Theta$ is non-Gaussian.

To illustrate the above theoretical results the case of $\eta=0.05, \alpha=1.1$ and $\omega=1$ is considered. The theoretical pdf of $\Theta$ and $\dot{\Theta}$ based on the exact solution of equation (6) for the case of $D_{11}=D_{22}=0.005$ and $D_{12}=0$ is shown in Fig. 3. The results for $D_{11}=0.005$, $D_{22}=0.0025$ and $D_{12}=0.0$ based on the non-linearization solution of equation (11) is shown in Fig. 4. In these figures the results of digital simulations are also shown. In the simulation studies, equation (2) has been integrated from $t=0$ to $t=500(2 \pi / \omega) \mathrm{s}$. The first 50 cycles of the response have been discarded to account for the transients. With the remaining sample solution, the pdf of the response has been estimated under the assumption of ergodicity. The response standard deviation obtained using the theory and the simulation respectively are also shown in Figs 3 and 4.
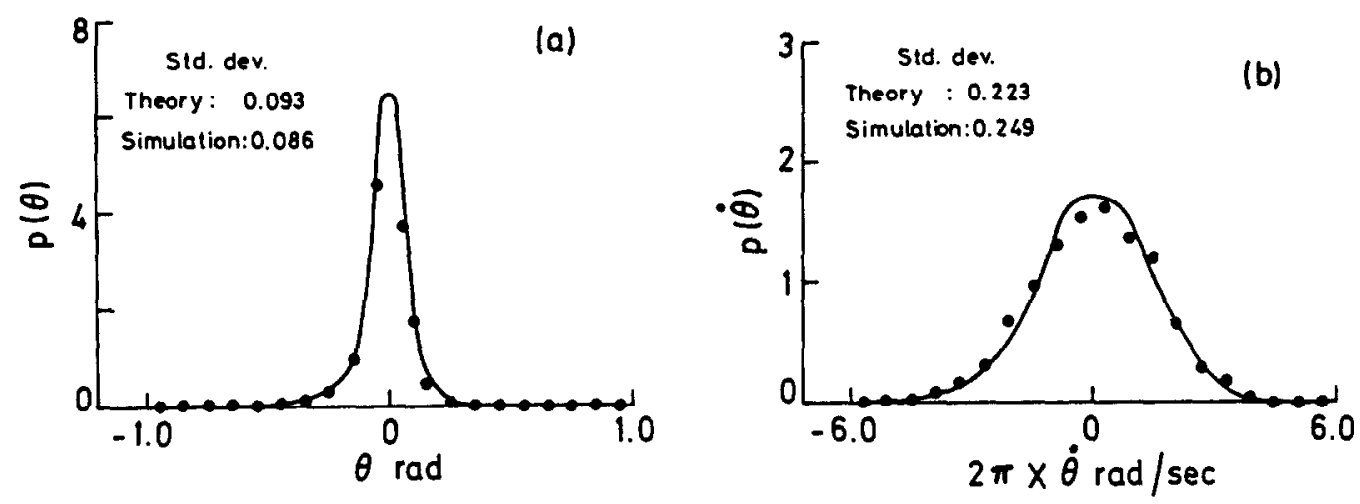

Fig. 3. Response probability density function; $D_{11}=D_{22}=0.005, D_{12}=0, \eta=0.05, \omega=1, x=1.1$. - theory; simulation. 

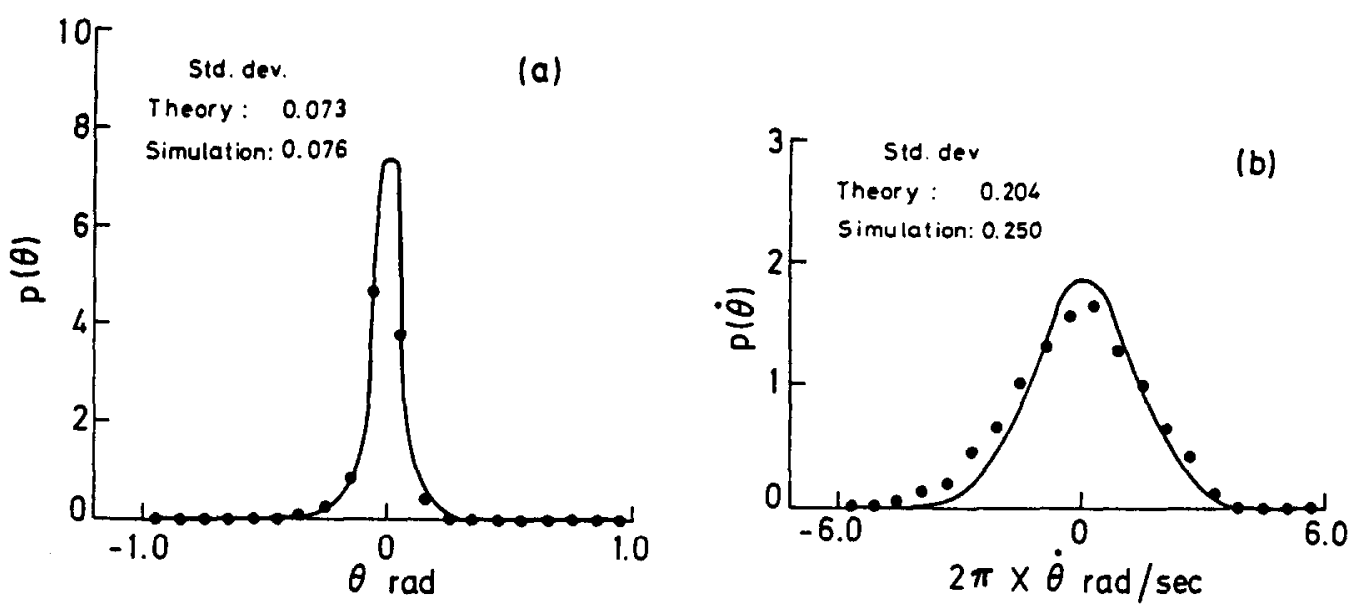

Fig. 4. Response probability density function; $D_{11}=0.005, D_{22}=0.0025, D_{12}=0, \eta=0.05, \omega=1$, $\alpha=1.1$. - theory; $\bullet$ simulation.

\section{OVERTURNING OF THE BLOCK}

The analysis presented in the previous section is based on the assumption that the block does not overturn while rocking, and that the kind of stochastic steady-state assumed in the analysis exists. In practice, however, the response trajectories over a long run of time can be expected to cross the critical response levels leading to the toppling of the block. If one allows this possibility into the analysis, the solution of equation (4) will have to be obtained in conjunction with an absorbing barrier placed along a suitably defined critical response level. Alternatively, one can study this as a first passage problem. Here one typically obtains the probability density function or the moments of the time required for the block to reach the critical level as a function of the initial position in the phase plane. Let $\Omega$ denote the safe region in the phase plane. This means that if a trajectory exits from $\Omega$, it would automatically lead to the toppling of the block. Further, define the random variable $T(x, y)$ as the time required for a trajectory initiated at $\Theta\left(t_{0}\right)=x$ and $\dot{\Theta}\left(t_{0}\right)=y$ to exit from the safe region $\Omega$ for the first time. The moments of this random variable denoted by $M_{n}=\left\langle T^{n}\right\rangle$, satisfy the well known GPV equations [10] given by the equation

$$
L M_{n}+n M_{n-1}=0 \quad n=0,1,2, \ldots
$$

with $M_{0}=1$. Here $L$ is the backward Kolmogorov operator

$$
L=y \frac{\partial}{\partial x}-\left[2 \eta \omega y+\omega^{2} \sin (\alpha \operatorname{sgn} x-x)\right] \frac{\partial}{\partial y}+f(x) \frac{\partial^{2}}{\partial y^{2}} .
$$

The boundary condition for equation (15) is

$$
M_{n}(\Omega)=0 .
$$

It may be pointed out here that if overturning is to be taken as a failure, it may not be sufficient to take just the level $\Theta= \pm \alpha$ as the critical position. Even though under static considerations toppling is imminent at $\Theta= \pm \alpha$, it is clear that under the dynamic situation failure should depend on both $\Theta$ and $\Theta$. Here it seems appropriate to take the region bounded by the separatrix in the phase plane to be the safe region. However, since it is difficult to handle the analytical equation of the separatrix, for purposes of co-ordinate transformation the separatrix is approximated by the ellipse written as

$$
\left(x^{2} / \alpha^{2}\right)+\left[y^{2} /\left(\omega^{2} \beta^{2}\right)\right]=1
$$

Further with the transformations

$$
\begin{aligned}
& x=r \alpha \cos \psi \\
& y=\omega \beta r \sin \psi
\end{aligned}
$$


the safe region gets mapped onto a circle with unit radius. Equation $(15)$ in the $(r, \psi)$ co-ordinate system can now be written as

$$
\begin{aligned}
& \omega \beta r \sin \psi\left[\frac{\partial M_{n}}{\partial r}(\cos \psi / \alpha)+\frac{\partial M_{n}}{\partial \psi}(-\sin \psi / r \alpha)\right] \\
& \quad-\left\{2 \eta \omega^{2} \beta r \sin \psi+\omega^{2} \sin [\alpha \operatorname{sgn}(r \alpha \cos \psi)-r \alpha \cos \psi]\right\} \\
& \quad\left[\frac{\partial M_{n}}{\partial r}(\sin \psi / \omega \beta)+\frac{\partial M_{n}}{\partial \psi}(\cos \psi / r \omega \beta)\right] \\
& \quad+f(r \alpha \cos \psi)\left[\frac{\partial^{2} M_{n}}{\partial r^{2}}\left(\sin ^{2} \psi / \omega^{2} \beta^{2}\right)+2 \frac{\partial^{2} M_{n}}{\partial r \partial \psi}\left(\sin \psi \cos \psi / r \omega^{2} \beta^{2}\right)\right. \\
& \quad+\frac{\partial M_{n}}{\partial r}\left(\cos ^{2} \psi / r \omega^{2} \beta^{2}\right)+\frac{\partial^{2} M_{n}}{\partial \psi^{2}}\left(\cos ^{2} \psi / r^{2} \omega^{2} \beta^{2}\right) \\
& \left.\quad+\frac{\partial M_{n}}{\partial \psi}\left(-\sin 2 \psi / r^{2} \omega^{2} \beta^{2}\right)\right]+n M_{n-1}=0
\end{aligned}
$$

The appropriate boundary condition for this equation is

$$
M_{n}(1, \psi)=0 .
$$

The solution of equation (20) using Galerkin's technique can be obtained by assuming a series of solution of the form

$$
M_{n}(r, \psi)=T_{n}(r)+\sum_{m=1}^{\infty}\left[a_{n m}(r) \sin 2 \pi m \psi+b_{n m}(r) \cos 2 \pi m \psi\right] .
$$

This leads to a set of ordinary differential equations for the unknown coefficients $T_{n}, a_{n m}$ and $b_{n m}$ which further have to be solved under the condition of equation (21). If as a first approximation only one term is retained in the expansion, the equation for $T_{n}$ is obtained as

$$
Q_{2}(r) \frac{\mathrm{d}^{2} T_{n}}{\mathrm{~d} r^{2}}+Q_{1}(r) \frac{\mathrm{d} T_{n}}{\mathrm{~d} r}+n T_{n-1}=0
$$

Here

$$
T_{n}(1)=0
$$

$$
\begin{gathered}
Q_{1}=-\eta \omega r+\left[1 /\left(r \omega^{2} \beta^{2}\right)\right](1 / 2 \pi) \int_{0}^{2 \pi} f(r \alpha \cos \psi) \cos ^{2} \psi \mathrm{d} \psi \\
Q_{2}=\left[1 /\left(\omega^{2} \beta^{2}\right)\right](1 / 2 \pi) \int_{0}^{2 \pi} f(r \alpha \cos \psi) \sin ^{2} \psi \mathrm{d} \psi .
\end{gathered}
$$

Further introduce

$$
P_{n}=\frac{\mathrm{d} T_{n}}{\mathrm{~d} r}
$$

This, together with the condition $T_{n}(1)=0$ leads to

The governing equation for $P_{n}$ is

$$
T_{n}(r)=-\int_{r}^{1} P_{n}(s) \mathrm{d} s
$$

$$
\frac{\mathrm{d} P_{n}}{\mathrm{~d} r}+\left(Q_{1} / Q_{2}\right) P_{n}=-n T_{n-1} / Q_{2}
$$

The solution of this equation in general can be written as

$$
P_{n}(r)=\exp \left[-\int\left(Q_{1} / Q_{2}\right) \mathrm{d} r\right]\left\{-\int_{0}^{r} \exp \left[\int\left(Q_{1} / Q_{2}\right) \mathrm{d} s\right]\left(n T_{n-1} / Q_{2}\right) \mathrm{d} s+C_{n}\right\} .
$$


The constant of integration $C_{n}$ appearing in the above solution has to be evaluated to satisfy the condition $M_{n}(0)<\infty$. For illustration, the case of $n=1, D_{11}=D_{22}=D$ and $D_{12}=0$ is considered. Here one gets

$$
\begin{aligned}
& Q_{1}=-\eta \omega r+0.5 D \omega^{2} /\left(r \beta^{2}\right) \\
& Q_{2}=0.5 D \omega^{2} / \beta^{2} .
\end{aligned}
$$

Accordingly, equation (29) can be simplified as

$$
P_{1}(r)=[1 /(\eta \omega r)]+\left(C_{1} / r\right) \exp \left[r^{2} \eta \beta^{2} /(D \omega)\right] .
$$

The constant $C_{1}$ is now chosen to ensure $P_{1}(0)=0$. This leads to

$$
P_{1}(r)=[1 /(\eta \omega r)]\left\{1-\exp \left[r^{2} \eta \beta^{2} /(D \omega)\right]\right\} .
$$

Using the series expansion for the exponential term, one further gets

$$
P_{1}(r)=[1 /(\eta \omega)] \sum_{n=1}^{\infty}\left\{\left[\eta \beta^{2} /(D \omega)\right]^{n} r^{2 n-1} / n !\right\} .
$$

Combining this with equation (27), the mean first passage time is obtained as

$$
T_{1}(r)=[1 /(2 \eta \omega)] \sum_{n=1}^{\infty}\left\{\left[\eta \beta^{2} /(D \omega)\right]^{n}\left(1-r^{2 n}\right) /(n ! n)\right\}
$$

The numerical results based on the above result for the case of $r=0, \alpha=1.1, \omega=0.05$, and for different values of $D$ and $\omega$ are shown in Fig. 5. The summation appearing on the right-hand-side of the above equation was found to converge with fifteen terms. Results of digital simulations for the case of $\omega=1$ are also shown in this figure. In the simulation work, numerical integration of equation (2) for every sample of $W_{1}(t)$ and $W_{2}(t)$ was carried out till $|\Theta(t)|$ reached the value of $0.5 \pi$. The corresponding value of the time variable was recorded as the sample realization of the first passage time. The estimate of the mean first passage time shown in Fig. 5 has been obtained with a sample size of 500 .

\section{DISCUSSION AND CONCLUSIONS}

In this paper, the planar rocking of rectangular blocks under white-noise base excitations has been studied using Markov process techniques. For low levels of the noise wherein the toppling of the block is unlikely, the stationary solution of the governing Fokker-Planck equation for the response pdf has been obtained under the assumption of the existence of a stochastic steady state. The exact solution presented in equation (6) is valid only for a specific combination of system parameters, while the approximate solution based on the equivalent non-linearization technique given in equation (11) is more generally valid. It may

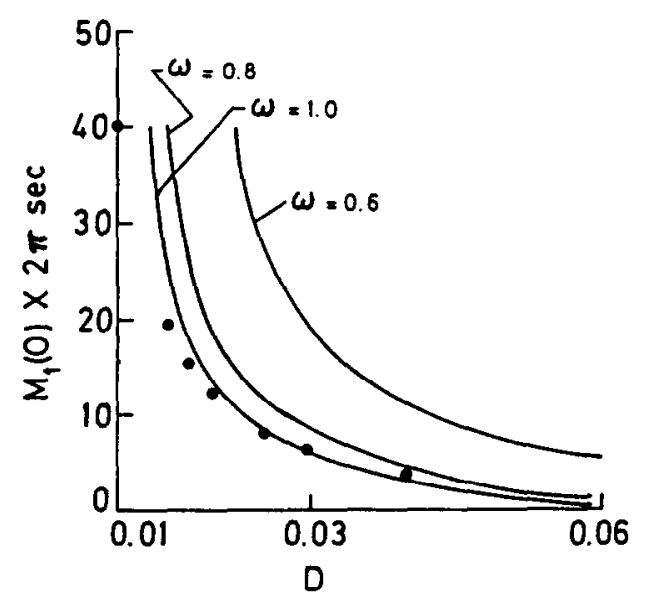

Fig. 5. Mean first passage time; $\eta=0.05, \alpha=1$.1. theory; $\bullet$ simulation for $\omega=1$. 
be noted that for the case of $D_{22} \rightarrow D_{11}$ and $D_{12} \rightarrow 0$, the non-linearization solution reduces to the exact solution given in equation (6). The theoretical response probability density functions are found to be in good agreement with the digital simulation results.

As the noise level is increased, the possibility of the toppling of the block becomes imminent and the assumption of the existence of a stochastic steady-state is no longer tenable. The problem of toppling of the block has been handled in the framework of first passage failure studies. In the analysis, the toppling of the block, or in other words, the failure of the system, is considered to have occurred when the response trajectory in the phase plane crosses the separatrix of the undamped free vibration of the system. A general expression for the $n$-th moment of the first passage time has been obtained using a one term Galerkin's series solution to the governing GPV equations. The preliminary numerical results on the mean first passage time for the specific case of $D_{11}=D_{22}$ and $D_{12}=0$ are found to compare favorably with the digital simulation results. It may be emphasized here that in the simulation work the block was considered to have toppled only when the rotation response reach the level $\pm 0.5 \pi$. The numerical results obtained support the choice of the separatrix of the unforced undamped motion as the critical barrier. For low levels of noise the theory is, however, found to overestimate the mean first passage time. The results are expected to improve with the inclusion of greater number of terms in the series solution given in equation (22). In this context it may be noted that as a consequence of retaining only one term in the series solution, the moments of the first passage time become axisymmetric in $r-\psi$ plane. Further work to incorporate the dependence of the moments on the $\psi$-co-ordinate by including additional terms in the expansion is currently being carried out by the authors.

A limitation of the present work has been the modeling of the dissipation of energy due to impacts through an equivalent viscous damping term. It must be noted that the inclusion of energy loss due to impacts into the equations of motion results in delta-function type of non-linearities which are difficult to treat analytically. However, it appears possible to employ a piecewise differentiable transformation on the dependent variable to overcome this difficulty. This aspect is also being currently investigated by the authors.

\section{REFERENCES}

1. M. Aslam, W. G. Godden and D. T. Scalise, Earthquake response of rigid bodies. J. Struct. Div. ASCE 106, 377 (1980).

2. C. S. Yim, A. K. Chopra and J. Penzien, Rocking response of rigid blocks to earthquakes. Earthquake Engng Struct. Dyn. 8, 565 (1980).

3. Y. Ishiyama, Motions of rigid bodies and criteria for overturning by earthquake excitations. Earthquake Engng Struct. Dyn. 10, 635 (1982).

4. P. D. Spanos and A. S. Koh, Rocking of rigid blocks due to harmonic shaking. J. engng Mech. Div. ASCE 110, 1627 (1984).

5. R. N. Iyengar, K. R. Reddy and C. S. Manohar, Chaotic response of some simple nonlinear oscillators. J. Aero. Soc. India 41, 1 (1990).

6. S. J. Hogan, On the dynamics of rigid-block motion under harmonic forcing. Proc. $R$. Soc. Lond. A425, 441 (1989).

7. P. D. Spanos and A. S. Koh, Analysis of block random rocking. Soil Dyn. Earthquake Engng 5, 178 (1986).

8. A. S. Koh, Rocking of rigid blocks on randomly shaking foundations. Nucl. Engng Des. 97,269 (1986).

9. T. K. Caughey, On the response of nonlinear oscillators to stochastic excitations. Prob. engng Mech. 1, 2 (1986).

10. J. B. Roberts, First-passage probabilities for randomly excited systems: Diffusion methods. Prob. engng Mech. $1,66(1986)$. 